Peiris, M.P.S.N. and De Silva, N., 2019. Fuzzy logic model to benchmark maintenance strategies for concrete structures. In: Sandanayake, Y.G., Gunatilake, S. and Waidyasekara, A. (eds). Proceedings of the $8^{\text {th }}$ World Construction Symposium, Colombo, Sri Lanka, 8-10 November 2019, pp. 360-367. DOI: doi.org/10.31705/WCS.2019.36. Available at: https://2019.ciobwcs.com/papers

\title{
FUZZY LOGIC MODEL TO BENCHMARK MAINTENANCE STRATEGIES FOR CONCRETE STRUCTURES
}

\author{
M.P.S.N. Peiris ${ }^{1}$ and Nayanthara De Silva ${ }^{2}$
}

\begin{abstract}
Maintenance of a building, which is of utmost importance, has become a burden to organisations worldwide, due to the unplanned approach towards it. Recently, views on building maintenance had undergone fundamental changes and are currently thought of as a crucial function in any organisation. Regrettably, around one-third of the allocated maintenance costs are wasted due to the ineffectiveness of maintenance planning. As a remedy to this loss, many organisations are currently shifting towards the incorporation of maintenance strategies such as corrective maintenance, preventive maintenance, and predictive maintenance. However, the implementation of these strategies itself will not solve the problem. Significant planning should be undergone in order to obtain the maximum benefit of executing these strategies. Introducing a tool to support planning and decision-making regarding maintenance strategy implementation will hence simplify this process. Therefore, the aim of this paper is to review existing literature on maintenance strategies and develop a fuzzy logic model to find the best combination of such maintenance strategies for concrete structures. Hence this paper portrays a conceptual model that can be adopted to benchmark maintenance strategies for concrete structures, by adapting the existing models which are commonly developed for maintenance of machinery and equipment.
\end{abstract}

Keywords: Benchmarking of Maintenance; Building Defects; Concrete Structures; Fuzzy Logic; Maintenance Strategies.

\section{INTRODUCTION}

Buildings are man-made structures used for providing shelter, protection and a platform for human activities (Hussain, 2016). Concrete structures, in specific, are the most common type of high-rise buildings, which is proven for its high durability and strength (Ali, 2001). However, its performance depends on the way it is designed, constructed and maintained (Dias, 2013). At present, many initiatives are researched to optimize design and construction practices of concrete structures aiming at obtaining highly durable and strong buildings (Ali, 2001). However, maintenance of concrete structures is undergone very poor maintenance practices, hence, contributing towards the faster deterioration of buildings (Dias, 2013).

Deterioration of concrete structures comes in the form of concrete defects such as dampness, cracks, water penetration, corrosion, etc. (Bakri and Mydin, 2012). According to the author, these defects undergo a severe process of internal deterioration before

\footnotetext{
${ }^{1}$ Department of Building Economics, University of Moratuwa, Sri Lanka, sandunipeiris95@gmail.com

2 Department of Building Economics, University of Moratuwa, Sri Lanka, endds@uom.lk
} 
appearing on the surface or any location visible. Therefore, it is vital to carry out maintenance at the early stage of the deterioration or even before starting the deterioration, in order to maintain its expected performance such as durability, integrity, strength, etc., of the structure.

Recent building maintenance records depict the sole usage of Corrective Maintenance (CM) strategy for concrete structures (Suffian, 2013). Maintenance activities in CM are done after the defect occurs, and thus is considered as unplanned maintenance. Therefore, it can be regarded as treating a wound to stop it from becoming worse (Deighton, 2016).

As a result, Preventive Maintenance (PM) and Predictive Maintenance (PdM) are introduced as planned strategies (Swanson, 2001). They can be applied to treat the root cause of the defects, thereby minimizing the damage to the structure. However, these are not commonly practiced in high rise concrete structures due to the extensive planning required and complexity (Olanrewaju et al., 2010). Therefore, researchers have suggested the use of unplanned (such as CM) and planned strategies in combination (Arruda, 2006; Pintelon et al., 2006; Ahuja and Khamba, 2008; Albarkoly and Park, 2015).

Therefore, it is vital to find the best combination of suitable maintenance strategies for the maintenance of concrete structures. Previous researchers have developed a Fuzzy Logic (FL) models mainly for maintenance planning of machinery (Al-Najjar and Alsyouf, 2003; Sharma et al., 2005). However, no such models have been developed for concrete structures. Hence, it is high time to develop a theoretical model to facilitate this purpose (Olanrewaju et al., 2010).

This research aims to develop a failure-cause based FL model for concrete structures enabling building managers to select the best combination of maintenance strategies for their buildings. This may contribute towards minimizing the negative impact of poor maintenance by utilizing the best combination of maintenance strategies at the initial stages itself.

\section{LITERATURE REVIEW}

\subsection{MAINTENANCE In CONCRETE STRUCTURES}

Maintenance in concrete structures mainly comes in the form of defects. The summary of various defects which are to be seen in these structures, as highlighted by several authors including Chong and Low (2006), Chew et al., (2004), Bakri and Mydin (2012), are shown in Table 1.

Table 1: Building elements and related defects

\begin{tabular}{ll}
\hline \multicolumn{1}{c}{$\begin{array}{c}\text { Building } \\
\text { Element }\end{array}$} & \multicolumn{1}{c}{ Defects } \\
\hline $\begin{array}{l}\text { Columns and } \\
\text { beams }\end{array}$ & Concrete crack, Plaster crack, stain, paint peeling, and blister \\
$\begin{array}{l}\text { Internal } \\
\text { Walls }\end{array}$ & $\begin{array}{l}\text { Plaster crack, stain, water seepage, paint peeling and blister, paint and plaster } \\
\text { patchy, chipped, concrete and tiles crack, other types of cracks, tiles falling/ } \\
\text { popping }\end{array}$ \\
$\begin{array}{l}\text { External } \\
\text { Walls }\end{array}$ & $\begin{array}{l}\text { Cracks, wall dampness, plaster crack, crazing, biological growth, staining, } \\
\text { paint peeling, paint crack, blistering, discoloration, chalking, efflorescence }\end{array}$
\end{tabular}




\begin{tabular}{ll}
\hline \multicolumn{1}{c}{$\begin{array}{c}\text { Building } \\
\text { Element }\end{array}$} & \multicolumn{1}{c}{ Defects } \\
\hline Roof & Leakage, cracks, waterproofing delaminating, stain \\
Floor & $\begin{array}{l}\text { Cracks, water seepage, tile delamination, unevenness, stains, hollowness, } \\
\text { discoloured tiles, efflorescence, chipped tiles }\end{array}$ \\
\hline
\end{tabular}

The objective of maintenance management is avoiding, diminishing and restoring building defects by improved preparation and execution using suitable resources at the correct time and lowest whole life-cycle cost (Tucker, 2007). With the aim of pursuing this objective, maintenance managers are currently considering cost-effective and reliable maintenance strategies to be implemented in organisations (Lee and Scott, 2009).

\subsection{MAINTENANCE STRATEgIES FOR CONCRETE STRUCTURES}

In maintaining a building/concrete structure there are typically numerous strategic options and different decisions to be considered by the management. Different authors have interpreted the options in different ways. However, out of the maintenance strategies available, the following three are commonly used (Horner et al., 1997; Begley, 2001; Lee and Scott, 2009; Lind and Muyingo, 2012; Bakri and Mydin, 2014).

\subsubsection{Corrective Maintenance}

Mostafa (2004) explains CM as unexpected activities carried out to bring the building back to its operating condition, or a building element to a condition that is fit for the intended purpose. According to the author, the main characteristic of CM is that actions are only completed after a failure. Hence, it causes several disadvantages such as higher downtime and overall maintenance costs (Sharma et al., 2005).

\subsubsection{Preventive Maintenance}

The aim of conducting PM is to minimise the recurrent and unexpected failures by performing maintenance activities such as repairs, testing, and inspection at a precise prearranged interval of time without considering the condition of the building (Garg and Deshmukh, 2006). Author further claims that the successful execution of PM and determination of time interval requires a decision support system.

\subsubsection{Predictive Maintenance}

The main outcome of PdM is deciding whether to maintain a building or not, according to its condition (Garg and Deshmukh, 2006). According to the author, this strategy lessens the chance of sudden failures with the help of diagnostics and timely interference. For example, diagnostic equipment is incorporated to measure the physical conditions such as crack propagation, corrosion, etc. to find out the root cause(s) and failure mechanisms (Chen and Trivedi, 2002).

However, among those, the most common type of maintenance strategy used in buildings/concrete structures is CM because it is the traditional method which is also simple in use (Lind and Muyingo, 2012). Planned PM is also used according to the authors. PdM is slightly used due to the complexity and the knowledge and experience required.

While many literature articles have been published on building maintenance and strategies to be adopted in implementing maintenance, effective use of these concepts 
cannot be achieved without suitable decision support systems or tools. Hence certain studies have considered maintenance strategy benchmarks to obtain better results.

\subsection{USE OF FUZZY LOGIC FOR BENCHMARKING OF MAINTENANCE STRATEGIES}

Literature found various studies that used FL in maintenance decision making. FL is identified as a strong methodology in transforming statements in natural language- verbal formation of the problem (such as level of maintenance required, optimised use of maintenance strategies) into a mathematical logic function called 'fuzzy numbers' (Sharma et al., 2005).

The studies discussed below have used FL to make maintenance-related decisions of machinery parts (See Table 2).

Table 2: Previous studies on FL based maintenance strategy selection

\begin{tabular}{ll}
\multicolumn{1}{c}{ Source } & Year \\
\hline Sharma et al. & 2005 \\
Al-Najjar and Alsyouf & 2003 \\
Mechefske and Wang & 2001 \\
\hline
\end{tabular}

Mechefske and Wang (2001) have used FL to attain biased assessments of maintenance strategies to obtain unbiased maintenance decisions for defects of centrifugal and reciprocal compressors. Al-Najjar and Alsyouf (2003) have used FL to develop a model to select the most effective maintenance strategy for machinery and equipment. This model was established using fuzzy logic functions derived based on a number of machine defects associated with rolling element bearings. Later in 2015, Sankpal et al. (2015) have developed a similar model for maintenance strategy selection of machine parts using a risk-based approach. Thus, this defect-oriented maintenance strategy model (refer Figure 1) could be used as an effective tool to analyse the importance of different failure causes in machines and the best combination of maintenance strategies to overcome them.

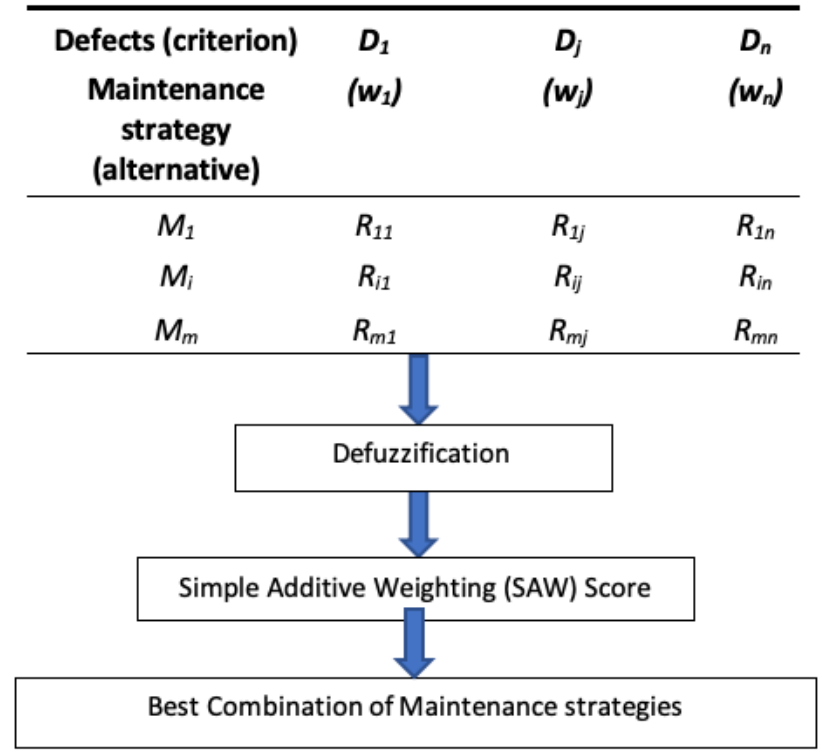

Figure 1: Defect-oriented maintenance strategy model 
$\mathrm{D}_{\mathrm{j}} \quad \mathrm{j}^{\text {th }}$ Defect

$\mathrm{M}_{i} \quad=i^{\text {th }}$ Maintenance Strategy

' $m$ ' = Number of maintenance strategies

' $n$ ' $\quad=$ Number of defects (criteria) in the plant

$w \quad=\left\{w_{j}\right.$, for $\left.j=1,2, \ldots, n\right\} \rightarrow$ fuzzy numbers indicating weights/importance of the criteria

$R \quad=\left\{R_{i j}\right.$, for $\left.i=1,2, \ldots, m ; \mathrm{j}=1,2, \ldots, n\right\} \rightarrow$ fuzzy numbers rating capability of the

maintenance strategy $(M)$ on identifying changes in the criterion

The model in Figure 1 uses defects of a particular machine (e.g. temperature, vibration) indicated by ' $\mathrm{D}_{j}$ ' as the criteria for benchmarking maintenance strategies for this machine. Each defect is given a weight ' $w_{j}$ ' in fuzzy numbers to indicate its impact on machine deterioration (e.g. impact of high temperature (criterion) on machine deterioration). Each maintenance strategy $\left(\mathrm{M}_{i}\right)$ is ranked against each defect using a fuzzy number ' $R_{i j}$ ' indicating the capability of $\mathrm{M}_{i}$ on identifying changes in $\mathrm{D}_{j}$ (e.g. how well can CM detect high temperature variations inside a machine?) The fuzzy ' $R$ ' values and fuzzy ' $w$ ' values are then defuzzified to obtain meaningful numbers. These defuzzified numbers are then ranked using Simple Additive Weighting (SAW) and a score $\left(\mathrm{S}_{\mathrm{i}}\right)$ is established for each maintenance strategy as given in equation (01).

$$
S_{i}=\sum W_{j} R_{i j}
$$

where, $\mathrm{S}_{i}=$ scores; $W_{j}=$ weight of $j^{\text {th }}$ defect; and $R_{i j}=$ effectiveness of $i^{\text {th }}$ maintenance strategy in rectifying the $j^{\text {th }}$ defect

These scores are finally normalised to obtain percentages of each maintenance strategy which can be used as a benchmark

\section{RESEARCH METHOD}

As discussed above, a defect-oriented maintenance strategy model developed using FL is identified as an effective tool for maintenance decision making in machinery. Thus, in this research, the defect-oriented maintenance strategy model developed originally by AlNajjar and Alsyouf (2003) is modified to develop a 'concrete defect-oriented maintenance strategy (CDMS)' model to benchmark maintenance strategies established for concrete structures.

Since the concrete structures are formed using a number of elements, separate CDMS models were developed to analyse maintenance needs and the best combination of maintenance strategies for each element. Figure 2 illustrates the CDMS model developed for the structural element 'roof' of a building.

Four types of defects including leakages, cracks, waterproofing delamination and stains are selected as defects $(n=4)$ in roofs. The weightages $(w)$ for those defects are derived based on the impact of those defects to the deterioration of the concrete structure. In this regard, six factors such as (1) impact on safety and health of building occupants, (2) frequency of occurrence, (3) cost of rectification, (4) impact on building performance, (5) ease of rectification of the defects, and (6) tendency to form another defect' are used to assess the impact (Das and Chew, 2011; Chew and De Silva, 2004). The ratings obtained for these factors (in terms of high, medium, low) are fed into the model using FL membership functions to obtain weightages $(w)$ in fuzzy numbers. 


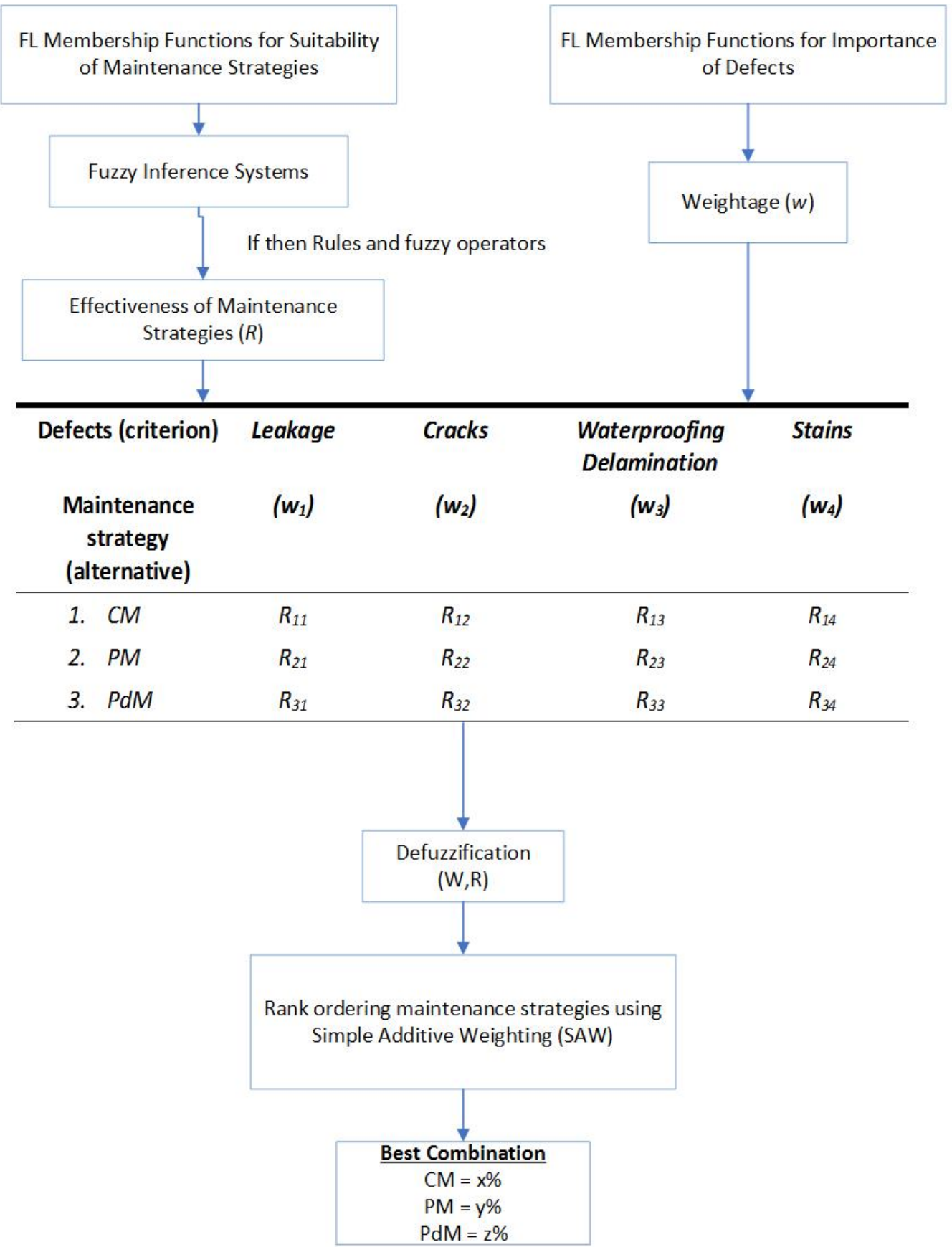

Figure 2: Concrete Defect-oriented Maintenance Strategy (CDMS) model for roof element

Three maintenance strategies (most commonly used) such as CM, PM and PdM are selected to benchmark the maintenance strategies (i.e. best combination of maintenance strategies). Their suitability to rectify each defect is assessed based on four factors; (1) value-added to element, (2) maintenance cost, (3) execution capability, and (4) impact on safety and health of occupants (Bevilacqua and Braglia, 2000; Wang et al., 2006; Azizi and Fathi, 2014). Assessment ratings obtained (in terms of high, medium, low) are then fed into the model using FL membership functions. Using if-then rules and fuzzy operators, a fuzzy inference system converts the assessment results to obtain ' $R$ ' values in fuzzy numbers. The interpretation of 'R' by Al-Najjar and Alsyouf (2003) for machines has been modified in the CDMS to suit the context of buildings. Therefore in the proposed 
CDMS ' $R$ ' value indicates the effectiveness of each maintenance strategy in rectifying each defect. For example, $R_{11}$ in Figure 2 is a fuzzy number that indicates how effective $\mathrm{CM}$ is in rectifying leakages.

In the next step, all fuzzy numbers are defuzzified in order to convert fuzzy numbers into real numbers that can be understood by humans. Since all ' $w$ ' values and ' $R$ ' values are in fuzzy numbers, they are defuzzified for meaningful information.

Next, the scores $S_{i}$ are calculated using equation (01) and normalised to obtain the best combination of maintenance strategies for the structural element roof as illustrated in Figure 2. The percentages $\mathrm{x}, \mathrm{y}$, and $\mathrm{z}$ indicate the proportions of CM, PM, and PdM respectively required to obtain the best combination of maintenance strategies for roof maintenance.

\section{CONCLUSIONS}

The aim of this paper is to benchmark the maintenance strategies for concrete structures. Literature was collected and is presented on maintenance requirements and maintenance strategies used for concrete structures, an introduction to FL and its applications in maintenance strategy benchmarking. A conceptual model is presented in the methodology as a tool for building managers.

The model presents a methodology that can be used to convert expert knowledge and judgements regarding concrete structure maintenance into solid, quantitative benchmarks. The model uses qualitative information such as the impact of building defects, level of maintenance required and effectiveness of maintenance strategies. The fuzzy operators are used to covert these qualitative data into fuzzy numbers to map the defects and the strategies based on suitability. These fuzzy numbers are then defuzzified and rank-ordered to establish the best proportion of maintenance strategies to be carried out on an element. The model in this paper is presented for the element 'roof'.

This model can be used by building managers to take the correct decision in the selection of maintenance strategies. This can be considered as a novel concept that can be used as a maintenance planning tool by the building managers while enhancing the attention towards maintenance of concrete structures. It is also suggested that this model is tested for validity in further research.

\section{REFERENCES}

Ahuja, I. and Khamba, J., 2008. Total productive maintenance literature review and directions. International Journal of Quality \& Reliability Management, 25(7), pp.709-756.

Albarkoly, K. M. and Park, K. S., 2015. Implementing a strategy of reliability centered maintenance (RCM) in the Libyan cement industry. International Journal of Social, Behavioral, Educational, Economic, Business and Industrial Engineering, 9(6), pp.1903-1912.

Ali, M. M., 2001. Evolution of Concrete Skyscrapers: from Ingalls to Jin mao. Electronic Journal of Structural Engineering, 1(1), pp.2-14.

Al-Najjar, B. and Alsyouf, I., 2003. Selecting the most efficient maintenance approach using fuzzy multiple criteria decision making. International Journal of Production Economics, 84(1), pp.85-100.

Arruda, C. H., 2006. Maintenance Evaluation and Benchmarking, Chile: MAPLA.

Azizi, A. and Fathi, K., 2014. Selection of optimum maintenance strategies based on a fuzzy analytic hierarchy process. Management Science Letters, 4(5), pp.893-898.

Bakri, N. and Mydin, M., 2014. General building defects: causes, symptoms and remedial work. European Journal of Technology and Design, 3(1), pp.4-17. 
Begley, M., 2001. EPM FM1 - Maintenance Strategy.

Bevilacqua, M. and Braglia, M., 2000. The analytic hierarchy process applied to maintenance strategy selection. Reliability Engineering and System Safety,70(1), pp.71-83.

Chen, D. and Trivedi, K., 2002. Closed-form analytical results for condition-based maintenance. Reliability Engineering \& System Safety, 76(1), pp.43-51.

Chew, M., and De Silva, N., 2004. Factorial method for performance assessment of building facades. Journal of Construction Engineering and Management, 130(4), pp.525-533.

Chew, M. Y., De Silva, N. and Tan, S. S., 2004. A neural network approach to assessing building façade maintainability in the tropics. Construction Management and Economics, 22(6), pp.581-594.

Chong, W.-K. and Low, S.P., 2006. Latent building defects: causes and design strategies to prevent them. Journal of Performance of Constructed Facilities, 20(3), pp.213-221.

Das, S. and Chew, M., 2011. Generic method of grading building defects using FMECA to improve maintainability decisions. Journal of Performance of Constructed Facilities, 25(6), pp.522-533.

Deighton, M. G., 2016. Chapter 5 - Maintenance Management. In: Facility Integrity Management, pp.87139.

Dias, W.P.S., 2013. Factors influencing the service life of buildings. Engineer: Journal of the Institution of Engineers, Sri Lanka, 46(4), pp.1-7.

Garg, A. and Deshmukh, S. G., 2006. Maintenance management: literature review and directions. Journal of Quality in Maintenance Engineering, 12(3), pp.205-238.

Horner, R. M., El-Haram, M. A. and Munns, A. K., 1997. Building maintenance strategy: a new management approach. Journal of Quality in Maintenance Engineering, 3(4), pp.273-280.

Hussain, M., 2016. Built Environment and Health. IOSR Journal of Humanities and Social Science, pp.16.

Olanrewaju, A.L., Khamidi, M.F. and Idrus, A., 2010. Building maintenance management in a Malaysian university campuses: a case study. Australasian Journal of Construction Economics and Building, 10(1/2), p.101.

Lee, H. H. Y. and Scott, D., 2009. Overview of maintenance strategy, acceptable maintenance standard and resources from a building maintenance operation perspective. Journal of Building Appraisal, 4(4), pp.269-278.

Lind, H. and Muyingo, H., 2012. Building maintenance strategies: planning under uncertainty. Property Management, 30(1), pp.14-28.

Mechefske, C. and Wang, Z., 2001. Using fuzzy linguistics to select optimum maintenance and condition monitoring strategies. Mechanical Systems and Signal Processing, 15(6), pp.1129-1140.

Mostafa, S. I., 2004. Implementation of proactive maintenance in the Egyptian Glass Company. Journal of Quality in Maintenance Engineering, 10(2), pp.107-122.

Mydin, M., 2015. Significance of building maintenance management system towards sustainable development: A review. Journal of Engineering Studies and Research, 21(1), pp.58-65.

Pintelon, L., Pinjala, S. K. and Vereecke, A., 2006. Evaluating the effectiveness of maintenance strategies. Journal of Quality in Maintenance Engineering, 12(1), pp.7-20.

Sankpal, P., Andrew, A. and Kumanan, S., 2015. Maintenance Strategies selection using Fuzzy FMEA and Integer Programming. In Proceedings of the International Conference on Advances in Production and Industrial Engineering (p. 503).

Sharma, R. K., Kumar, D. and Kumar, P., 2005. FLM to select suitable maintenance strategy in process industries using MISO model. Journal of Quality in Maintenance Engineering, 11(4), pp.359-374.

Suffian, A., 2013. Some common maintenance problems and building defects: Our experiences. Procedia Engineering, 54, pp.101-108.

Swanson, L., 2001. Linking maintenance strategies to performance. International Journal of Production Economics, 70(3), pp.237-244.

Tucker, M., 2007. Building Maintenance Management, Blackwell Publishing Ltd.

Wang, L., Chu, J. and Wu, J., 2006. Selection of optimum maintenance strategies based on a fuzzy analytic hierarchy process. International Journal of Production Economics, 107(1), pp.151-163. 